\title{
Factores explicativos del éxito competitivo: el caso de las PyMEs del estado de Veracruz
}

\author{
Antonio Aragón Sánchez \\ Alicia Rubio Bañón *
}

\section{Resumen}

Las investigaciones que estudian el origen de las diferencias de competitividad entre organizaciones muestran que se deben fundamentalmente a las características estructurales del entorno industrial en el que se desarrolla la competencia entre empresas (efecto industria) y a las variables que configuran el ámbito interno de las organizaciones (efecto empresa). Además, el análisis de la importancia relativa de dichos efectos muestra que el segundo es más importante para explicar el logro de ventajas competitivas.

Con este trabajo se trata de conocer cuáles son los factores internos sobre los que las PYME articulan su ventaja competitiva, utilizando para ello una muestra de 1.425 pequeñas y medianas empresas del estado de Veracruz. Los resultados que se encontraron son consistentes con los recogidos en la bibliografia al destacar la importancia que para el éxito tienen las capacidades financieras, la posición tecnológica, la innovación, las capacidades de marketing, la dirección y gestión de los recursos humanos, así como las tecnologías de la información y comunicación.

Palabras clave: competitividad, PYME, ventaja competitiva, recursos y capacidades.

\footnotetext{
* Profesores-Investigadores de la Universidad de Murcia, España. Correos electrónicos: aaragón@um.es; arubio@um.es
} 


\section{INTRODUCCIÓN}

Cómo alcanzar y sostener la ventaja competitiva es una cuestión crucial para el éxito y la supervivencia de las empresas. El estudio de las causas de las diferencias de rentabilidad entre empresas es un tema controvertido y ampliamente tratado en la literatura académica dado que son muchos los trabajos que han buscado responder a la pregunta: ¿cuáles son las variables que explican la competitividad de la empresa?

En una primera fase, la investigación se centró en el análisis de la influencia de variables relacionadas con los entornos general y específico; posteriormente, a comienzos de los años ochenta, los trabajos en esta materia se reorientan al mostrar que, si bien las variables externas influyen en el logro de una ventaja competitiva, es mucho mayor la incidencia de las variables internas. Estos resultados supusieron un cambio importante en la concepción de los elementos explicativos de la competitividad y de los resultados empresariales, aportando un sólido apoyo empírico a la teoría de recursos y capacidades como marco de análisis de las diferencias de competitividad entre organizaciones.

Desde este enfoque cada empresa es única debido, principalmente, a los recursos que posee y a la forma en que los gestiona; su capacidad para competir dependerá, por tanto, fundamentalmente de ella misma. Que la empresa cuente con una gran cantidad de recursos y que sean numerosas las posibilidades para su gestión, lleva a los investigadores a plantearse la necesidad de estudiar con más detenimiento la parte interna de las organizaciones para tratar de determinar cuáles son realmente los factores que explican la ventaja competitiva.

Un análisis de la bibliografía muestra que, si bien son muchos los trabajos que analizan la importancia de las variables externas frente a las internas, son escasos los que profundizan en la parte interna para identificar cuáles son las variables que la empresa debe asegurar o aplicar para ser competitiva, representando aspectos claves a los que hay que prestar especial atención si se quieren conseguir resultados superiores (Jennings y Beaver, 1997; Rogoff et al., 2004). Estamos ante un tema que entraña gran complejidad tanto por la falta de consenso existente en la literatura acerca de la unidad de análisis o del marco teórico por adoptar como por la dificultad que presenta la elección de una definición clara y operativa de competitividad.

Esta escasez de trabajos es aún mayor si acotamos el análisis a las empresas de menor tamaño. La importancia que las PYME tienen en la economía, por su gran 
contribución a la creación de empleo y al progreso económico y social, no se ve reflejada en el número de investigaciones que se centren en su estudio. Esto justifica, dadas las especificidades o competencias distintivas que tienen, la realización de estudios que identifiquen los factores de éxito propios de las PYME (Jennings y Beaver, 1997).

Esta situación nos ha llevado a tratar de clarificar dos cuestiones: ¿qué se entiende por competitividad? y ¿qué factores internos la propician en las empresas de menor tamaño? La respuesta a estas cuestiones permitirá avanzar en el ámbito de la dirección estratégica y en el conocimiento científico y práctico de las PYME.

Para la consecución de estos objetivos, el presente trabajo se estructura en torno a tres apartados. En el primero de ellos se establece el marco teórico que delimita el concepto de competitividad y que establece un modelo de gestión que se centra en los factores de éxito competitivo en las PYME que nos lleva a plantear las hipótesis de investigación. En seguida, se contrasta el modelo empíricamente apoyándonos en una muestra de 1.544 PYME representativas del conjunto de este tipo de empresas en el estado de Veracruz. Por último, se ofrecen las implicaciones que los resultados obtenidos tienen para la investigación de este fenómeno.

\section{MARCO TEÓRICO}

Son muchas y muy diversas las propuestas que pueden encontrarse en la bibliografía relativas a qué se entiende por éxito competitivo o competitividad empresarial. ${ }^{1}$ La mayor parte de las definiciones coinciden en definir la competitividad como una capacidad para generar ventajas competitivas sostenibles, para producir bienes y servicios creando valor o para actuar ante la rivalidad suscitada al relacionarse con otra empresa.

En este trabajo se entiende por la competitividad empresarial o éxito competitivo de una empresa la capacidad para, rivalizando con otras empresas, conseguir alcanzar una posición competitiva favorable, que permita obtener un desempeño superior al de los competidores. ${ }^{1}$ Este concepto lo definen, entre otros, Kester y Luerhrman (1989), Porter (1990), Viedma (1992),
Álvarez y García (1996) y Camisón (1997). 
Como se observa, la definición propuesta es amplia y teórica; para delimitarla con una mayor precisión, siguiendo a Venkatraman y Ramanujam (1986), a continuación se revisa cómo diversas investigaciones empíricas han conceptualizado y operativizado el término (tabla 1). De dicha revisión se concluye que la rentabilidad económica, pese a los inconvenientes que presenta, es la medida más utilizada por los investigadores.

Este hecho plantea dos nuevas cuestiones: ¿es correcto medir el éxito competitivo a través de una única medida? y ¿cuál es la fuente de obtención de datos más apropiada? 
Factores explicativos del éxito competitivo: el caso de las PYME del estado de Veracruz

\section{Tabla 1: Indicadores de medida del éxito competitivo}

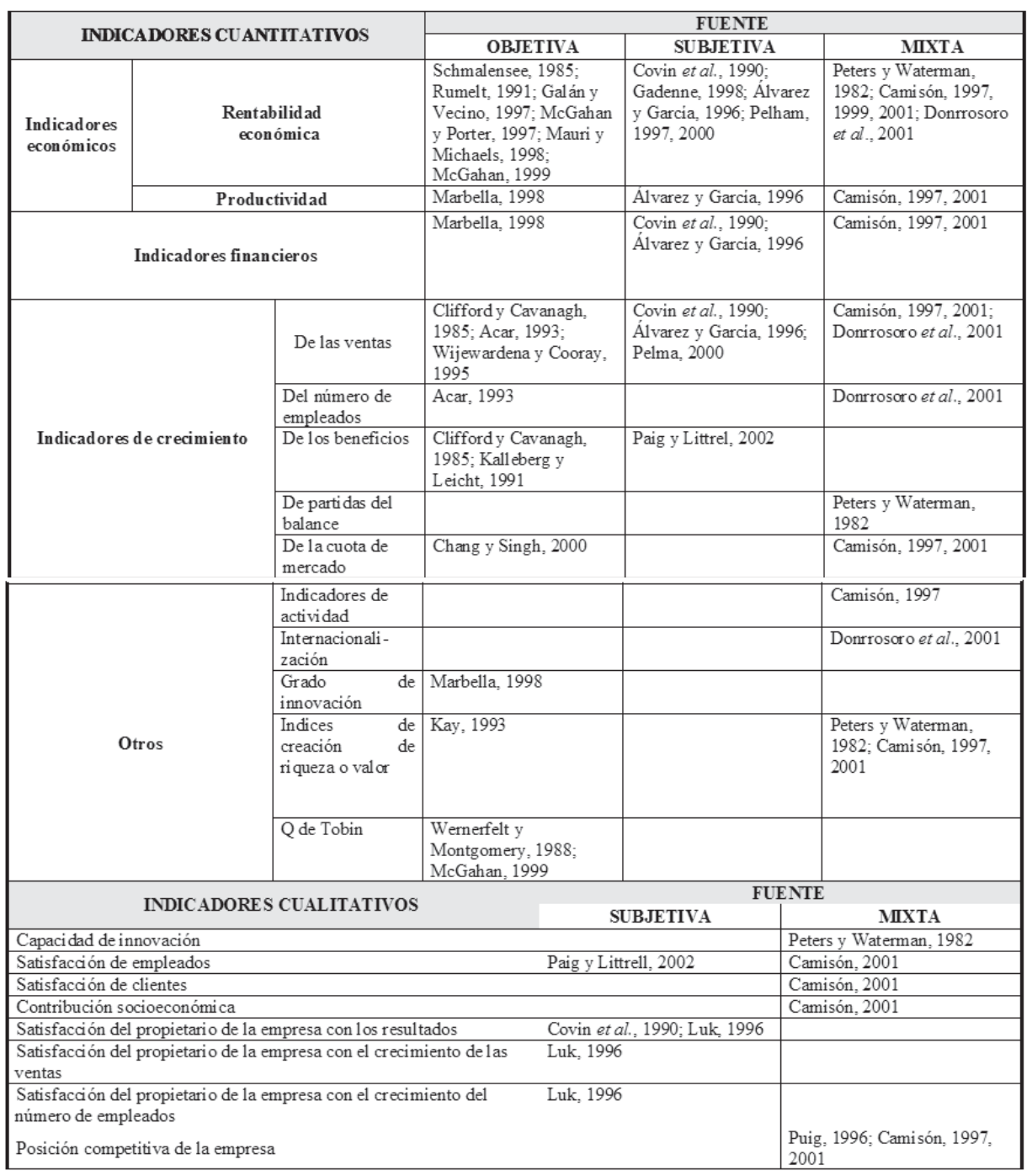

Fuente: Elaboración propia 
Con respecto a la primera cuestión, se considera más idónea la utilización de una medida multidimensional de naturaleza cuantitativa y cualitativa debido a que la naturaleza de la competitividad se ve afectada por un gran número de variables de naturaleza tanto tangible como intangible, imposibles de reflejar en una medida unidimensional como es la rentabilidad económica (Daily y Dalton, 1992:28; Kotey y Meredith, 1997).

En cuanto a la segunda cuestión, la literatura ha seguido diversos medios para identificar y medir el desempeño organizativo (tabla 1). Concretamente, según la fuente de información utilizada para obtener los datos, se pueden distinguir entre fuentes objetivas $^{2}$ y subjetivas $^{3}$ (Camisón, 1999).

A pesar de no existir un claro consenso en el tipo de fuente de información por utilizar, en este trabajo se propone la utilización de fuentes subjetivas por diferentes motivos.

En primer lugar, las características de la información empresarial, basada en el sistema de información contable, introduce una peculiar forma de registrar y clasificar impregnada de una serie de consideraciones legales y fiscales que sesgan su objetividad al alejar el auténtico valor de la empresa y sus auténticos rendimientos (Varadajan y Ramanujan, 1990:469). Por otro lado, las empresas son muy reticentes a aportar este tipo de datos (Covin et al., 1990: 492; Pelham, 1997:57), y de las bases de datos existentes no siempre se obtienen datos fiables. Además, las fuentes subjetivas de desempeño son más útiles cuando se utilizan muestras de PYME (Covin et al., 1990:492); este tipo de empresas pueden ver reducidas sus cifras contables temporalmente por inversiones en desarrollo de nuevos productos, tecnologías o procesos sin que esto indique una peor posición competitiva.

En segundo lugar, de la definición propuesta de éxito competitivo se deduce que la medida por utilizar debe proporcionar información sobre la posición relativa de la empresa con respecto a su competencia. Por tanto, es más adecuado medir cada una de las variables mediante una escala que permita conocer dicha posición.

${ }^{2}$ Evaluaciones del éxito competitivo que no están sujetas a las percepciones de los individuos, sino que se fundamentan en indicadores objetivos obtenidos mediante estudio de casos, encuestas, bases de datos, etc. (Clifford y Cavanagh, 1985; Acar, 1993; Kay, 1994).

${ }^{3}$ Evaluaciones del éxito competitivo a través de las percepciones que realizan individuos (Conant et al., 1990; Lin, 1998; Gadenne, 1998). Se trata, pues, de autovaloraciones. 
La utilización de una escala multidimensional, que incluya tanto variables cuantitativas como cualitativas, y el uso de fuentes subjetivas nos van a permitir una medida más precisa del éxito competitivo, especialmente en las PYME.

\section{Factores explicativos del éxito competitivo}

El segundo objetivo de este trabajo es determinar ¿cuáles son los factores internos que propician la competitividad en las PYME?

Para ello, se parte de los resultados de la corriente de investigación que, encabezada por Rumelt (1991), sostiene que la aptitud de la empresa para obtener resultados superiores depende fundamentalmente de su habilidad para adquirir y coordinar recursos situando al efecto empresa y al marco teórico que lo sustenta, la teoría de recursos y capacidades, en el centro del análisis de la competitividad empresarial.

Para conocer los recursos y capacidades estratégicos de las PYME, se ha efectuado una revisión de diversos trabajos que se centran en el estudio de la trayectoria seguida por las PYME de éxito con el objetivo de establecer las principales coincidencias entre ellos.

Dado que cada una de las investigaciones adopta su propio lenguaje y dota de sus propios significados a los términos y conceptos utilizados, es preciso establecer un marco integrador que va a ser, de acuerdo con Grant (1996), la teoría de recursos y capacidades.

Utilizar esta teoría es consistente con el enfoque sostenido por numerosos trabajos como los de Roquebert et al., 1996; Mauri y Michaels, 1998; y Camisón, 2001. Es posible afirmar que la capacidad de la empresa para obtener éxito en los mercados dependerá fundamentalmente de los recursos que posea y de la forma en que son gestionados.

Las principales coincidencias de los trabajos que analizan las PYME (tabla 2) evidencian que las ventajas competitivas de éstas se sustentan en la adquisición y gestión de sus recursos tangibles, intangibles y humanos, así como de sus capacidades.

Los trabajos revisados muestran que el mantenimiento de buenos estándares financieros, a través de una adecuada gestión financiera, es uno de los principales 
factores resaltados como necesarios para alcanzar el éxito competitivo en las PYME. En este sentido, es necesario realizar con cautela la planificación a corto plazo, implantar y controlar sistemas de contabilidad de costos, prestar una especial atención a las entidades de crédito, establecer presupuestos anuales, realizar análisis de la situación económico-financiera e intentar, en la medida de lo posible, utilizar fuentes de financiación propias (Birley y Westhead, 1990:554). De ahí se desprende que:

$H_{1}$ : El éxito competitivo de las PYME se ve influido positivamente por las capacidades financieras de la empresa.

Tabla 2. Principales factores de éxito competitivo

\begin{tabular}{|c|c|c|}
\hline & Factor resaltado & Autores \\
\hline $\begin{array}{l}\text { RECURSOS } \\
\text { TANGIBLES }\end{array}$ & $\begin{array}{c}\text { Adecuada gestión } \\
\text { financiera }\end{array}$ & $\begin{array}{l}\text { Birley y Westhead, 1990; Huck y McEwen, 1991; V iedma, 1992; Acar, } \\
\text { 1993; Yusuf, 1995; Álvarez y García, 1996; Gadenne, 1998; Warren y } \\
\text { Hutchinson, 2000. }\end{array}$ \\
\hline \multirow{4}{*}{$\begin{array}{l}\text { RECURSOS } \\
\text { INT ANGIBLES }\end{array}$} & Recursos tecnológicos & Álvarez y García, 1996; Donrrosoro et al., 2001. \\
\hline & Innovación & $\begin{array}{l}\text { Viedma, 1992; Puig, 1996; Camelo et al., 1999; Donrrosoro et al., 2001; } \\
\text { Pil y Holwelg, 2003; Ribeiro, } 2003 .\end{array}$ \\
\hline & $\begin{array}{c}\text { Capacidades de } \\
\text { marketing }\end{array}$ & $\begin{array}{l}\text { Clifford y Cavanagh, 1985; Huck y McEwen, 1991; Viedma, 1992; } \\
\text { Álvarez y García, 1996; Luk, 1996; Pelham,. 1997; Lin, 1998; Camelo et } \\
\text { al., 1999; Warren y Hutchinson, 2000; Donrrosoro et al., 2001; Pelma, } \\
\text { 2000; Pil y Holwelg, 2003; Verhees y Meulenberg, 2004. }\end{array}$ \\
\hline & Calidad & Luk, 1996; Camelo et al., 1999; Donrrosoro et al., 2001. \\
\hline $\begin{array}{l}\text { RECURSOS } \\
\text { HUMANOS }\end{array}$ & $\begin{array}{l}\text { Políticas y procesos de } \\
\text { dirección de recursos } \\
\text { humanos }\end{array}$ & $\begin{array}{l}\text { Clifford y Cavanagh, 1985; Kirby, 1990; Huck y McEwen, 1991; } \\
\text { Viedma, 1992; Vinckerstaff, 1992; Pfeffer, 1994; Yusuf, 1995; } \\
\text { Wijewardena y Cooray, 1995; Luk, 1996; Puig, 1996; Gadenne, 1998; } \\
\text { Lin, 1998; Camelo et al., 1999; Loan-Clarke et al., 1999; Smith et al., } \\
\text { 1999, Llopis, 2000; McLarty, 2000; Santos y González, 2000; V inten, } \\
\text { 2000; Warren y Hutchinson, 2000; Donrrosoro et al., 2001, Huang, } 2001 .\end{array}$ \\
\hline CAPACIDADES & $\begin{array}{l}\text { Capacidades directivas } \\
\text { Sistemas de información }\end{array}$ & $\begin{array}{l}\text { Dollinguer, 1984; Norburn y Birley, 1988; Huck y McEwen, 1991; } \\
\text { Viedma, 1992; Acar, 1993; Yusuf, 1995; Ahiere y Golhar, 1996; Luk, } \\
\text { 1996; Puig, 1996; Camisón, 1997; Lin, 1998; European Foundation for } \\
\text { Quality Management, 2000; Ottewill et al, 2000; Donrrosoro et al., } \\
\text { 2001. } \\
\text { Viedma, 1992; Llopis, 2000; Donrrosoro et al., } 2001 .\end{array}$ \\
\hline
\end{tabular}

Fuente: Elaboración propia

En segundo lugar están los recursos tecnológicos y las inversiones en tecnología de producto y/o producción. La capacidad de la empresa para producir bienes y servicios depende de sus activos tecnológicos; por tanto, la tecnología adquirida por la empresa o el uso que de ella se haga determinará la posición con respecto a 
la competencia (Donrrosoro et al., 2001). Aquellas empresas que sólo realizan nuevas inversiones cuando comprueban que los competidores obtienen buenos resultados - comportamiento reactivo - o las que no posean información fiable de la posición tecnológica de las empresas rivales están avocadas al fracaso. Por ello, formulamos la siguiente hipótesis:

$\mathrm{H}_{2}$ : El éxito competitivo de las PYME se ve influido positivamente por la posición tecnológica de la empresa.

Un factor en el que coinciden muy diversos estudios por su clara contribución a la mejora de los resultados organizacionales es la innovación. El éxito descansa en la capacidad de la empresa para adaptarse mejor a los entornos dinámicos y complejos; es por ello que las empresas que quieran ser competitivas deberán afrontar los constantes cambios en las tecnologías, en los productos y servicios, así como en las preferencias de los consumidores, estableciendo los mecanismos que fomenten la innovación tanto en el nivel de producto como de proceso productivo y de gestión (Puig, 1996; Camelo et al., 1999; Donrrosoro et al., 2001; Pil y Holwelg, 2003; Ribeiro, 2003). Así la tercera hipótesis se formula de la siguiente manera:

$H_{3}$ : El éxito competitivo de las PYME se ve influido positivamente por el desarrollo de innovaciones.

La cuarta de las características resaltada por la literatura es la relacionada con las capacidades de marketing. La empresa de éxito se distingue de sus competidoras por una clara orientación al mercado y, sobre todo, por presentar una clara orientación hacia el cliente, las que son, si acaso, más importantes en las empresas de menor tamaño debido a que la mayor proximidad al cliente les permite un mejor y más rápido conocimiento de sus necesidades y de los medios para satisfacerlas (Pelham, 1997 y 2000; Pil y Holwelg, 2003:35; Verhees y Meulenberg, 2004).

Por tanto, aquellas empresas que sean capaces de desarrollar productos nuevos, que adapten los existentes a las necesidades de sus clientes y que generen valor al consumidor a través de la creación de imagen de marca tendrán una mayor capacidad para competir con otras empresas, lo que nos lleva a enunciar la cuarta de las hipótesis de este trabajo:

$H_{4}$ : El éxito competitivo de la empresa se ve influido positivamente por las capacidades de marketing. 
En quinto lugar, las PYME de éxito se caracterizan, además, por tratar de lograr la conformidad del conjunto de características y atributos del producto o servicio con las necesidades y expectativas del comprador. La calidad es otro de los factores que la literatura reconoce como explicativo de la competitividad por influir decisivamente en la imagen de la empresa en el mercado (Luk, 1996; Camelo et al., 1999; Donrrosoro et al., 2001). Aunque a corto plazo contar con productos y servicios de calidad puede suponer fuertes inversiones, a medio y largo plazo se reducen costos, se consiguen clientes satisfechos y se mejoran los resultados. Por ello, se plantea la siguiente hipótesis:

$H_{5}:$ El éxito competitivo de las PYME se ve influido positivamente por la calidad del producto o servicio.

El factor donde se observa una mayor coincidencia en la literatura como explicativo del éxito es el relativo a los recursos humanos de la empresa.

Así, los conocimientos, las habilidades y actitudes de los trabajadores y cómo trabajan éstos, son aspectos cada vez más importantes para las empresas debido a que las fuentes tradicionales de éxito son cada vez menos relevantes (Pfeffer, 1994).

Diversos autores coinciden en afirmar que el éxito de las PYME viene de la mano de las personas y, por lo tanto, es necesario contar con adecuadas políticas de gestión y dirección de los recursos humanos que promuevan la atracción del personal competente, la motivación a través de las políticas retributivas y una adecuada gestión de los planes formativos para lograr que el personal esté lo suficientemente calificado para afrontar el cambio organizacional y la creciente competencia. Esto nos lleva a proponer la siguiente hipótesis de investigación:

$H_{6}$ : El éxito competitivo de las PYME se ve influido positivamente por la aplicación de políticas de dirección de recursos humanos.

Un factor que también se identifica claramente en la literatura como explicativo de la competitividad de las PYME es la capacidad directiva para gestionar la empresa. En un intento por acercarnos al perfil ideal del directivo, la revisión realizada permite afirmar que las PYME deben estar dirigidas por personas que se adapten con facilidad a nuevas situaciones, procesen grandes volúmenes de información, sean capaces de gestionar sus empresas en entornos inciertos y ambiguos y se orienten a la creatividad e innovación (Dollinguer, 1984; Huck y McEwen, 1991; 
Acar, 1993; Yusuf, 1995; Luk, 1996; Lin, 1998; Ottewill et al., 2000; Donrrosoro et al., 2001).

El nivel de educación, al ser un reflejo de los conocimientos y habilidades poseídos, se relaciona de forma positiva con la capacidad del directivo para realizar elecciones estratégicas de acuerdo con las exigencias del entorno (Wiersema y Bantel, 1992), con su propensión a generar e implantar soluciones creativas a los problemas de la empresa (Bantel y Jackson, 1989) e incluso con el mayor nivel de productividad (Norburn y Birley, 1988).

Esto nos sugiere que las empresas que cuenten con directivos con un mayor nivel de capacitación alcanzarán mayores niveles de éxito. Como consecuencia, se deriva la siguiente hipótesis:

$H_{7}$ : El éxito competitivo de las PYME se ve influido positivamente por el nivel de formación del máximo responsable o gerente.

Asimismo, la literatura muestra que la incorporación de nuevos sistemas y tecnologías de la información propicia el éxito competitivo de la empresa. Las PYME deben desarrollar sistemas de información rápidos, sencillos, transparentes y prácticos porque éstos permiten afrontar la incertidumbre del entorno y fomentan la creatividad e innovación. Del mismo modo, la incorporación de nuevos sistemas y tecnologías de la información facilita la planificación, impulsa el desarrollo y ejecución de los programas de producción y estimula el control de los procesos y productos (Huerta et al., 2003). Por tanto, fomentar el uso de las nuevas tecnologías de la información y comunicación para la gestión de la empresa facilita el éxito competitivo (Viedma, 1992; Llopis, 2000; Donrrosoro et al., 2001). Esto nos lleva a plantear la última de las hipótesis de investigación:

$H_{8}$ : El éxito competitivo de las PYME se ve influido positivamente por las tecnologías de la información y comunicación en la empresa.

\section{METODOLOGÍA}

Tomando como referencia el marco teórico anterior, en este apartado se muestra la metodología empleada para contrastar las hipótesis, partiendo de los datos relativos a la obtención de la muestra de empresas, se continúa con la medida de las 
variables que se utilizan en el análisis empírico y se realiza una caracterización general de las PYME estudiadas.

\section{Población, muestra y obtención de datos}

La población seleccionada para el contraste de las hipótesis está formada por empresas del estado de Veracruz con más de cinco trabajadores y menos de 250. Dicha población para los efectos del diseño muestral ha sido segmentada de acuerdo con los criterios de actividad, región y tamaño. El número de empresas en cada uno de los estratos se ha obtenido cruzando diversas bases de datos disponibles, trabajando con una población total de 2.439 PYME. Cabe señalar que el diseño general de la muestra se fundamenta en los principios del muestreo estratificado en poblaciones finitas. ${ }^{4}$

Dado que el cuestionario incluye una gran variedad de tipos de preguntas y de variables - caracterización de la empresa, aspectos de dirección, de recursos humanos, de estructura organizativa, de tecnología, de innovación, de calidad, el equipamiento de comunicaciones y de medios informáticos, así como aspectos contables y financieros que son cuantitativas y cualitativas con dos o más respuestas-, el tamaño muestral se ha determinado para lograr que el margen de error máximo para la estimación de una proporción fuese, con un nivel de confianza del $95 \%$, inferior a 0,02 puntos para el total de la encuesta.

La recopilación de la información se ha llevado a cabo mediante la realización de una entrevista personal, estructurada con preguntas cerradas, al gerente de las PYME. El trabajo de campo se realizó entre los meses de abril a septiembre de 2003; una vez depurada la base de datos se trabajó con una muestra total de 1425 PYME. $^{5}$

\footnotetext{
${ }^{4}$ La muestra ha sido diseñada con el objetivo de que sea representativa de la estructura empresarial del conjunto del estado de Veracruz de acuerdo con los criterios de actividad, comarca y tamaño. Los sectores considerados han sido manufacturas, construcción, transporte, comercio y servicios y turismo. Por su parte, la clasificación regional ha sido la siguiente: Coatzacoalcos-Minatitlán, Veracruz, Poza RicaTuxpan, Xalapa y Córdoba-Orizaba. La variable tamaño se ha definido en función del número de empleados generándose dos grupos: microempresas (hasta 10 trabajadores) y pequeñas y medianas empresas (de 10 a 250 trabajadores)

${ }^{5}$ Las entrevistas a todas las empresas de la muestra habrían sido imposibles de realizar sin la desinteresada colaboración del equipo de coordinadores y encuestadores de la Universidad Veracruzana de sus distintos campus (Poza Rica-Tuxpan, Veracruz, Xalapa, Coatzacoalcos-Minatitlán y Córdoba-Orizaba) a los que desde aquí expresamos el más profundo agradecimiento.
} 


\section{Medición de las variables}

Para el contraste de hipótesis es preciso contar con datos relativos a los factores de éxito (variables independientes), a los factores explicativos del éxito y a las variables de control.

\section{Variables independientes}

Para medir todas las variables independientes, con la excepción de las capacidades financieras, se utilizan medidas cualitativas de percepción del directivo entrevistado.

Capacidades financieras: escala compuesta por tres ítemes relativos a: 1) la implantación y control de un sistema de contabilidad de costos, 2) el establecimiento de presupuestos de ingresos y gastos anual y 3 ) el análisis de la situación económicofinanciera. ${ }^{6}$ Una vez contrastada la validez de constructo en su faceta discriminante, a través de un análisis factorial, y su fiabilidad, a través del alfa de Cronbach $(0,859)^{7}$, se procedió a definir la variable como la suma de las tres anteriores; es decir, se miden las capacidades financieras en una escala de 5 hasta 15.

Posición tecnológica: inicialmente la medición de la posición tecnológica de las empresas se realizó utilizando una variable nominal de cuatro categorías dependiendo de que la empresa posea una posición tecnológica fuerte - desarrollo interno de la tecnología que utilizamos con el fin de obtener mejores resultados que la competencia - buena - la tecnología adquirida por la empresa o el uso que se hace de ella nos posiciona por delante de la competencia-, sostenible - la tecnología que utilizamos es la misma que se utiliza en la mayoría de empresas del sector y sólo realizamos nuevas inversiones cuando comprobamos que la competencia obtiene buenos resultados - o débil nuestros principales competidores tienen una tecnología más eficiente o moderna que la nuestra.

\footnotetext{
${ }^{6}$ Cada una de las variables se midió a través de una escala de diferencial semántico que varía desde $1=$ el nivel de utilización de la técnica es mínimo, hasta $5=$ el nivel de utilización de la técnica es máximo.

${ }^{7}$ Si la fiabilidad de las dimensiones está por encima de 0,6 se considera aceptable, de acuerdo con Hair et al. (1999:105-106). Igualmente, es posible aceptar aquellas escalas que superen el 0,5 si se trata de nuevas variables (Churchill, 1979).
} 
La necesidad de contar con variables continuas o dicotómicas nos ha llevado a transformarla en una variable dummy que toma el valor 1 cuando la empresa tiene una posición fuerte-buena y el valor 0 cuando ésta es sostenible-débil.

Innovación: para medir el nivel de innovación se pidió a las PYME que señalasen las áreas de su empresa donde se han efectuado innovaciones en los dos últimos años. Esto permitió la construcción de una variable suma que recoge el número de áreas en las que la empresa ha realizado innovaciones variando desde 0 hasta 3.

Capacidades de marketing: escala compuesta por tres ítemes: 1) acceso a nuevos mercados, 2) servicio al cliente y 3 ) habilidades y esfuerzos en actividades de mercadotecnia. ${ }^{8}$ Una vez contrastada la validez de constructo en su faceta discriminante - a través de un análisis factorial — y su fiabilidad, a través del alfa de Cronbach $(0,587)^{9}$, se ha construido una variable suma que valora las capacidades de marketing en una escala desde 5 hasta 15.

Calidad del producto o servicio: para conocer en qué medida las empresas gestionan la calidad de sus productos o servicios se ha preguntado a las empresas por el aseguramiento de la calidad. Concretamente se preguntó si la empresa disponía de certificación ISO de la serie 9000 o equivalente. Esto nos ha permitido construir una variable dummy que toma el valor 1 cuando la empresa cuenta con alguna certificación ISO de la serie 9000 o equivalente, o bien si se encuentra en el proceso de certificación, y toma el valor 0 cuando no lo está.

Dirección y gestión de los recursos humanos: para medir la dirección y gestión de los recursos humanos se pidió a las PYME que señalasen los procesos de recursos humanos que aplican en su empresa. Esto ha permitido la construcción de una variable suma que recoge el total de prácticas de recursos humanos implantadas en las PYME variando éste desde 0 hasta 5 (reclutamiento y selección, valoración del rendimiento/desempeño, formación, promoción del personal, implantación de sistemas de pago variable).

\footnotetext{
${ }^{8}$ Cada una de las variables se midió a través de una escala de diferencial semántico que varía desde $1=$ el factor no es nada importante para el desarrollo y éxito de la empresa, hasta $5=$ el factor es muy importante para el desarrollo y éxito de la empresa.

${ }^{9} \mathrm{Si}$ la fiabilidad de las dimensiones está por encima de 0,6 se considera aceptable, de acuerdo con Hair et al. (1999:105-106). Igualmente, es posible aceptar aquellas escalas que superen el 0,5 si se trata de nuevas variables (Churchill, 1979).
} 
Formación del gerente: toma el valor 1 cuando el gerente de la empresa tiene estudios universitarios y el valor 0 cuando éstos son primarios o de grado medio (bachillerato o formación profesional).

\section{Intensidad de las tecnologías de la información y comunicación en la em-} presa: es una variable cuantitativa que agrega las respuestas afirmativas a las siguientes cuestiones sobre la tenencia y uso de las siguientes tecnologías de la información y comunicación:I) correo electrónico (e-mail), II) página web, III) mantenimiento de relaciones con proveedores a través de internet, IV) realización de acciones de mercadotecnia a través de internet, v) línea telefónica básica, vI) líneas de infovía, internet, VII) fax, VIII) videotex/ibertex, IX) módem, X) ordenadores en red. Esta variable varía desde 0 hasta 10.

\section{Variables dependientes}

De las distintas medidas de éxito utilizadas por la literatura empírica, se ha decidido utilizar para esta investigación un indicador global de éxito, tanto por las limitaciones en la clasificación y registro de los datos contables para tener un indicador cuantitativo de resultados como por las ventajas de la utilización de una escala multidimensional que incluye tanto variables cuantitativas como cualitativas, lo que nos permitirá una medida más precisa y enriquecida del éxito competitivo.

Dicho indicador muestra la posición de la empresa en relación con sus competidores en las variables: I) cifra media de utilidades antes de intereses e impuestos, II) calidad de los productos o servicios, III) introducción de innovaciones, IV) productividad de la mano de obra, v) satisfacción de los clientes con los productos o servicios, VI) conocimiento y experiencia en el negocio, VII) motivación/satisfacción de los empleados y VIII) reputación e imagen de la empresa. Cada uno de los ítemes se midió con una escala tipo Likert que va desde (1) mucho peor que la competencia hasta (5) mucho mejor que la competencia. Para confirmar la fiabilidad de esta escala se ha calculado el coeficiente alfa de Cronbach obteniéndose un valor de 0,844 .

Nuevamente, para confirmar la validez del concepto en su faceta discriminante se ha recurrido al análisis factorial según el método de componentes principales con rotación ortogonal Varimax. Con este método se obtiene un único factor, cuyo 
valor propio fue superior a uno para las ocho dimensiones del éxito competitivo; por lo tanto, es representativo de cada una de las dimensiones introducidas. ${ }^{10}$

Teniendo en cuenta que la escala es fiable y válida, se ha construido una nueva variable suma que valora el éxito competitivo de la empresa en una escala que varía desde 8 hasta 40 . Una empresa exitosa obtendrá puntuaciones cercanas al valor 40 .

\section{Variables de control}

Para no desvirtuar el análisis de los datos y evitar posibles problemas de interpretación de los resultados, se han introducido las variables de control que según la bibliografía revisada pueden tener mayores efectos en el éxito competitivo de la empresa (Lafuente y Yagüe, 1989; Acar, 1993; Wijewardena y Cooray, 1995; Gadenne, 1998; Pelham, 2000; Santos y González, 2000; Reid et al., 2000; Caggliano et al., 2001; Camisón y Boronat, 2003):

- El tamaño, medido a través de una variable dummy que toma el valor 0 cuando la empresa tiene menos de 10 trabajadores (microempresa) y 1 cuando la empresa tiene entre 11 y 250 trabajadores (tamaño pequeño o mediano).

- Sector de actividad, utilizando para su medida una variable dicotómica que toma el 0 cuando la empresa pertenece al sector servicios y 1 si pertenece a la industria.

- La antigüedad de la empresa (años transcurridos desde su constitución).

- El tipo de control, medido a través de una variable dummy que toma el valor 1 cuando el control mayoritario de la empresa es familiar y 0 cuando no lo es.

- La estrategia, medida a través de una variable dummy que toma el valor 1 cuando la empresa adopta una estrategia exploradora y 0 en caso contrario. ${ }^{11}$

\footnotetext{
${ }^{10}$ Además el resultado de la prueba KMO con respecto a la adecuación muestral es alto $(0,831)$. Finalmente, los resultados del contraste de esfericidad de Barlett también indican la idoneidad del análisis realizado. ${ }^{11}$ La dinámica del entorno en el que actualmente se mueven las empresa mexicanas nos lleva a pensar que sólo aquellas empresas que realicen cambios y mejoras en los productos/servicios, conforme a los mercados, con relativa frecuencia y aquellas que traten de ser las primeras en desarrollar nuevos productos y servicios, es decir, las empresas que sigan una estrategia exploradora, estarán en el camino al éxito.
} 


\section{Caracterización de las empresas de la muestra}

Antes de proceder al contraste de las hipótesis, se hace un breve análisis descriptivo de cuáles son las principales características de las empresas de la muestra; para ello se utilizarán tablas de contingencia ${ }^{12}$ y análisis de la varianza. ${ }^{13}$

Se estudian cada una de las variables introducidas en los análisis: tamaño, sector, tipo de control, antigüedad y tipo de estrategia — variables de control—; y capacidades financieras, posición tecnológica, innovación, capacidades de marketing, calidad del producto y servicio, dirección y gestión de los recursos humanos, formación del gerente y tecnologías de la información y comunicación — variables independientes.

Concretamente, en el cuadro 1 se presentan los resultados correspondientes al tamaño, sector, tipo de control y antigüedad. Como se observa, estamos ante una muestra formada mayoritariamente por microempresas (57,2\%); en un $68,8 \%$ de los casos desarrollan su actividad en el sector servicios; pueden calificarse como jóvenes al tener una antigüedad inferior de 10 años en el 60,9\% de los casos; y son controladas mayoritariamente por un grupo familiar, $78,3 \%$.

\section{Cuadro 1: Caracterización de las PYME del estado de Veracruz}

\begin{tabular}{|c|c|c|c|c|c|c|c|}
\hline \multicolumn{2}{|c|}{ Tamaño } & \multicolumn{2}{|c|}{ Sector } & \multicolumn{2}{|c|}{ Tipo de Control } & \multicolumn{2}{|c|}{ Antigüiedad } \\
\hline Micro & $57,2 \%$ & Industria & $31,2 \%$ & Familiar & $78,3 \%$ & $\leq$ de 10 años & $60,9 \%$ \\
\hline $\begin{array}{l}\text { Pequeña/ } \\
\text { Mediana }\end{array}$ & $42,8 \%$ & Servicios & $68,8 \%$ & No familiar & $21,7 \%$ & $>$ de 10 años & $39,1 \%$ \\
\hline
\end{tabular}

Si nos centramos en el análisis de las variables independientes del modelo, cuadro 2, se observa que son las empresas más antiguas y las de mayor tamaño las que muestran una mayor orientación a la innovación.

\footnotetext{
${ }^{12}$ Cuando analizamos diferencias porcentuales se utiliza el análisis de tablas de contingencia a las que se les ha aplicado el test de la $\mathrm{c}^{2}$ de Pearson con el fin de valorar si dos variables se encuentran relacionadas. ${ }^{13}$ En el caso de variables donde se han aplicado diferencias de medias se utiliza el análisis de la varianza (ANOVA).
} 
A esta misma caracterización responden aquellas empresas con un mayor desarrollo de la capacidad para gestionar tanto las capacidades financieros como las tecnologías de la información y comunicación. Además, los datos muestran que las empresas no familiares son las que mantienen mejores estándares financieros y fomentan en mayor medida la utilización de los sistemas de información (cuadro 2).

Con relación a las capacidades de marketing, las PYME que presentan una mayor orientación al mercado y al cliente son las de mayor tamaño y las no familiares.

Los resultados obtenidos para las variables relativas al número de procesos de dirección y gestión de los recursos humanos que aplica la empresa muestran que nuevamente son las PYME más antiguas y de mayor tamaño las que utilizan un mayor número de procesos de recursos humanos. Por el contrario, en esta ocasión son las empresas controladas por un grupo familiar son las que utilizan un mayor número de procesos de gestión de recursos humanos, a diferencia de los que se venía observando con el resto de variables analizadas.

\section{Cuadro 2: Variables independientes según características de las empresa}

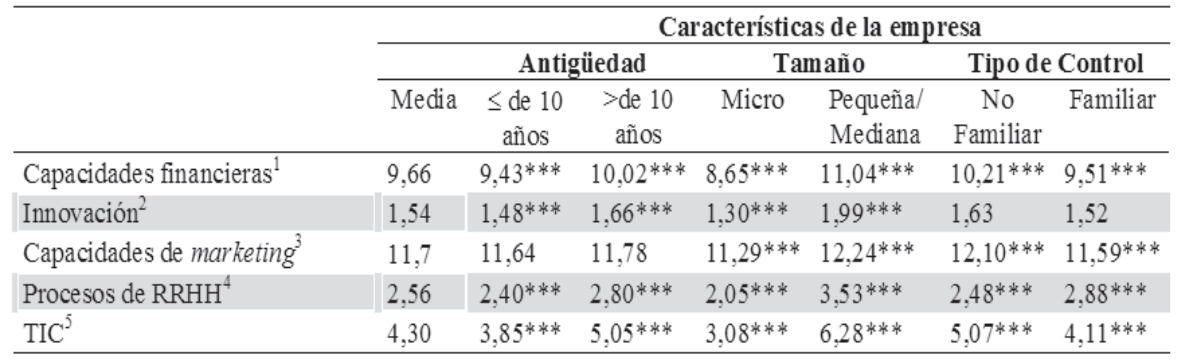

(1) En una escala de 5-15; (2) En una escala de 0-3; (3) En una escala de 5-15; (4) En una escala de 05; (5) En una escala de $0-10$.

$(*): \mathrm{p}<0,1 ;(* *): \mathrm{p}<0,05 ;(* * *): \mathrm{p}<0,01$

Por otro lado, el cuadro 3 muestra los resultados del análisis realizado con las variables tipo de estrategia, posición tecnológica, calidad y estudios del gerente.

Con respecto a la estrategia, los resultados ponen de manifiesto que el $41,5 \%$ de las PYME adoptan una estrategia defensiva, es decir, ofrecen un conjunto estable de productos y no están interesadas en la modificación de los mismos, sino que se 
concentran en la mejora continua del trabajo en su área de actuación; el 33,3\% siguen una estrategia analizadora, es decir, buscan el equilibrio entre el mantenimiento de los productos actuales y la introducción selectiva de nuevos productos; el $23,9 \%$ restante tienen una estrategia exploradora, lo que significa que tratan de ser los primeros del mercado introduciendo cambios y mejoras sustanciales tanto en los productos como en los mercados en los que operan.

Por otro lado, el análisis de las diferencias con respecto a la antigüedad, el tamaño y el tipo de control muestra como tan sólo se han encontrado diferencias en el tamaño. Son las empresas más grandes las que en mayor porcentaje siguen una estrategia exploradora, mientras que las microempresas adoptan mayoritariamente una estrategia defensiva.

Los resultados obtenidos para la variable posición tecnológica revelan que las empresas cuya posición tecnológica es fuerte-buena se caracterizan por ser más antiguas, tener un mayor tamaño y por no estar controladas por un grupo familiar.

\section{Cuadro 3: Variables independientes y estrategia según características de la empresa}

\begin{tabular}{|c|c|c|c|c|c|c|c|}
\hline & \multirow[b]{3}{*}{$\%$} & \multicolumn{6}{|c|}{ Características de la empresa } \\
\hline & & \multicolumn{2}{|c|}{ Antigüedad } & \multicolumn{2}{|c|}{ Tamaño } & \multicolumn{2}{|c|}{ Tipo de Control } \\
\hline & & $\begin{array}{c}\leq \text { de } 10 \\
\text { años }\end{array}$ & $\begin{array}{c}>\text { de } 10 \\
\text { años }\end{array}$ & Micro & $\begin{array}{l}\text { Pequeña/ } \\
\text { Mediana }\end{array}$ & $\begin{array}{c}\text { No } \\
\text { Familiar }\end{array}$ & Familiar \\
\hline \multicolumn{8}{|l|}{ ESTRATEGIA } \\
\hline * Exploradora & 25,3 & 23,9 & 27,3 & $21,1 * * *$ & $31,8 * * *$ & 26,3 & 25,2 \\
\hline * Analizadora & 33,7 & 33,3 & 34,4 & $31,4 * * *$ & $36,7 * * *$ & 34,7 & 33,3 \\
\hline * Defensiva & 41,0 & 42,7 & 38,3 & $47,5 * * *$ & $31,5 * * *$ & 39,1 & 41,5 \\
\hline \multicolumn{8}{|l|}{ P. TECNOLÓGICA } \\
\hline * Sostenible-débil & 49,5 & $51,6 * *$ & $46,4^{* *}$ & $58,5^{* * * *}$ & $34,5 * * *$ & $43,3 * *$ & $50,8 * *$ \\
\hline * Fuerte-buena & 50,5 & $48,4 * *$ & $53,6 * *$ & $41,5 * * *$ & $65,5 * * *$ & $56,7 * *$ & $49,2 * *$ \\
\hline \multicolumn{8}{|l|}{ CALIDAD } \\
\hline * No certificadas & 70,7 & $73,2 * * *$ & $67,0 * * *$ & $78,9 * * *$ & $59,4 * * *$ & $61,8 * * *$ & $73,0 * * *$ \\
\hline * Certificada o en proceso & 29,3 & $26,8 * * *$ & $33,0 * * *$ & $21,1 * * *$ & $40,6 * * *$ & $38,2 * * *$ & $27,0 * * *$ \\
\hline \multicolumn{8}{|l|}{ FORMACIÓN GERENTE } \\
\hline * No universitarios & 41,1 & 42,5 & 39,2 & $51,3 * * *$ & $26,1 * * *$ & $34,7 * * *$ & $42,9 * * *$ \\
\hline * Universitarios & 58,9 & 57,5 & 60,8 & $48,7 * * *$ & $73,9 * * *$ & $65,3 * * *$ & $57,1 * * *$ \\
\hline
\end{tabular}

$(*): \mathrm{p}<0,1 ;(* *): \mathrm{p}<0,05 ;(* * *): \mathrm{p}<0,01$ 
En relación con la variable calidad del producto y servicio, hay un 29,3\% de empresas que cuentan con certificación de calidad o están en proceso para alcanzarla. Las empresas que en mayor medida están certificadas se encuentran entre las de mayor edad y tamaño, así como en el grupo de las no familiares; esto nos está indicando que las empresas más jóvenes, de menor tamaño y familiares evidencian ciertas dificultades con la certificación de calidad.

Por último, es importante resaltar que en casi el 60\% de las PYME de la muestra el gerente posee estudios universitarios, hecho que puede calificarse como un punto fuerte para el desarrollo futuro de este colectivo de empresas. Como se esperaría, este porcentaje asciende en las empresas de mayor tamaño $(73,9 \%)$ y en las que la propiedad y control no está en manos de una familia $(65,3 \%)$.

\section{ANÁLISIS DE DATOS Y RESULTADOS}

Para el contraste de las hipótesis propuestas se ha realizado una regresión multivariante, al igual que en los trabajos de Acar (1993), Wijewardena y Cooray (1995) y Gadenne (1998). Matemáticamente la ecuación de regresión adopta la siguiente expresión:

$$
Y_{i j}=\beta_{o}+\sum_{k=1}^{5} \beta_{k} C_{k j}+\beta_{6} C F_{j}+\beta_{7} P T_{j}+\beta_{8} I_{j}+\beta_{9} C M_{j}+\beta_{10} C P S_{j}+\beta_{11} R R H H_{j}+\beta_{12} F G_{j}+\beta_{13} T I C_{j}+e_{i}
$$

donde:

$\mathbf{Y}_{\mathrm{j}}=$ valor del indicador de $\mathrm{j}$ (indicador global).

$\boldsymbol{\beta}_{0}=$ constante de la recta de regresión.

$\beta_{1}-\beta_{13}=$ parámetros de los coeficientes de variación parcial para cada una de las variables independientes. Representan la estimación del cambio en la variación de la variable dependiente que puede atribuirse a un incremento en una unidad en la variable independiente.

$\mathbf{E}_{\mathbf{i}}=$ error aleatorio o residuo de la recta de regresión. El residuo es la diferencia entre el valor real de la variable dependiente y el pronosticado por el modelo.

$\mathbf{C}_{\mathrm{kj}}=$ valor del indicador de control $\mathrm{k}$ para la empresa $\mathrm{j}(\mathrm{k}=1$, tamaño de la empresa; 2, sector; 3, tipo de control; 4, antigüedad; y 5, estrategia).

$\mathbf{C F}_{\mathbf{j}}=$ Capacidad financiera de la empresa $\mathrm{j}$.

$\mathbf{P T}_{\mathrm{j}}=$ Posición tecnológica de la empresa $\mathrm{j}$.

$\mathbf{I}_{\mathbf{j}}=$ Innovación en la empresa $\mathbf{j}$. 
$\mathbf{C M}_{\mathrm{j}}=$ Capacidades de marketing en la empresa $\mathrm{j}$

$\mathbf{C P S}_{\mathrm{i}}=$ Calidad del producto o servicio en la empresa $\mathrm{j}$.

$\mathbf{R R H H}_{\mathbf{j}}=$ Dirección y gestión de los recursos humanos en la empresa j.

$\mathbf{F G}_{\mathbf{j}}=$ Formación del gerente en la empresa $\mathbf{j}$.

$\mathbf{T I C}_{\mathbf{j}}=$ Tecnologías de la información y comunicación en la empresa $\mathbf{j}$.

Antes de analizar los resultados obtenidos se ha realizado un análisis de las correlaciones (cuadro 4) y de los índices de tolerancia, descartando con ello la multicolinealidad; asimismo, se comprobaron los supuestos exigidos para este tipo de análisis: linealidad, homocedasticidad, independencia y normalidad.

El cuadro 5 muestra los resultados de la regresión. Como se puede comprobar, el modelo es significativo alcanzando el $\mathrm{R}^{2}$ un valor del 0,395 . El poder explicativo del modelo es similar al obtenido por Wijewardena y Cooray (1995) y mayor que el obtenido en la investigación realizada por Acar (1993).

La inclusión de variables externas como el nivel de incertidumbre del entorno general, el atractivo del entorno específico, la pertenencia a un grupo estratégico o a un distrito industrial, sin duda, mejoraría el $\mathrm{R}^{2}$ del modelo, aunque en este trabajo no constituyen el objeto de estudio.

\section{Cuadro 4: Correlaciones}

\begin{tabular}{|c|c|c|c|c|c|c|c|c|c|c|c|c|c|}
\hline & IG & 1 & 2 & 3 & 4 & 5 & 6 & 7 & 8 & 9 & 10 & 11 & 12 \\
\hline 1.T & $0,157^{8 * 8 x}$ & & & & & & & & & & & & \\
\hline 2. Sector & $0,103^{* * *}$ & 0,040 & & & & & & & & & & & \\
\hline 3. C. & $-0,010$ & $-0,14^{* * *}$ & 0,045 & & & & & & & & & & \\
\hline 4. A. & $0,070^{* * * *}$ & $0,188^{* * *}$ & $-0,065^{* *}$ & 0,037 & & & & & & & & & \\
\hline 5. E & $0,186^{* * * 8}$ & $0,143^{* * *}$ & $-0,047$ & $-0,017$ & 0,051 & & & & & & & & \\
\hline 6. CF & $0,390^{* * 8 *}$ & $0,323^{* * *}$ & $-0,060^{* *}$ & $-0,07^{* * * 8}$ & $0,07^{* * *}$ & $0,233^{* 8}$ & & & & & & & \\
\hline 7. P.T. & $0,355^{* * *}$ & $0,238^{* * *}$ & $-0,023$ & $-0,06 * *$ & 0,050 & $0,271^{* *}$ & $0,351^{* *}$ & & & & & & \\
\hline 8.I & $0,360^{* * *}$ & $0,315^{* * *}$ & $0,110^{* * *}$ & $-0,044$ & $0,08^{* * *}$ & $0,204^{* *}$ & $\begin{array}{l}0,399 * * \\
z\end{array}$ & $0,360^{* *}$ & & & & & \\
\hline 9. $\mathrm{CM}$ & $0,514^{* * 8}$ & $0,186^{* * *}$ & $-0,028$ & $-0,08 * * *$ & 0,046 & $\begin{array}{l}0,177^{* *} \\
*\end{array}$ & $0,394^{* *}$ & $0,313^{* *}$ & $0,326^{* 8}$ & & & & \\
\hline 10. CPS & $0,136^{* * 8}$ & $0,212^{* * *}$ & 0,006 & $-0,10^{* * *}$ & $0,066^{* *}$ & $\begin{array}{l}0,173^{* *} \\
*\end{array}$ & $\begin{array}{l}0,194^{* *} \\
*\end{array}$ & $0,221^{* *}$ & $0,241^{* *}$ & $0,181^{* *}$ & & & \\
\hline $\begin{array}{l}11 . R R H \\
\mathrm{H}\end{array}$ & $0,332 * * *$ & $0,395^{* * *}$ & $-0,044$ & $-0,08^{* * *}$ & $0,11^{* * *}$ & $\begin{array}{l}0,216^{* *} \\
*\end{array}$ & $\begin{array}{l}0,474^{* *} \\
*\end{array}$ & $\begin{array}{l}0,342^{* *} \\
*\end{array}$ & $0,422^{* *}$ & $\begin{array}{l}0,336^{* *} \\
*\end{array}$ & $\begin{array}{l}0,236^{* *} \\
*\end{array}$ & & \\
\hline 12. FG & $0,085^{* * *}$ & $0,254^{* * *}$ & $-0,040$ & $-0,069^{* * *}$ & 0,033 & $\begin{array}{l}0,100^{* *} \\
*\end{array}$ & $\begin{array}{l}0,215^{* *} \\
{ }^{*}\end{array}$ & $\begin{array}{l}0,156^{* *} \\
*\end{array}$ & $\begin{array}{l}0,222^{* *} \\
*\end{array}$ & $\begin{array}{l}0,163^{* *} \\
*\end{array}$ & $\begin{array}{l}0,130^{* *} \\
*\end{array}$ & $\begin{array}{l}0,243^{* 8} \\
*\end{array}$ & \\
\hline 13. $\Pi \mathrm{CC}$ & $0,260^{* * *}$ & $0,517^{* * *}$ & $-0,053^{* *}$ & $-0,08 * * *$ & $0,20 * * *$ & ${ }_{*}^{0,163^{* *}}$ & $0,392^{* *}$ & ${ }_{*}^{0,353^{* *}}$ & ${ }_{*}^{0,409^{* *}}$ & $0,329^{* * *}$ & ${ }_{*}^{0,234^{* *}}$ & $0,453^{* *}$ & $0,370^{* *}$ \\
\hline
\end{tabular}

(*): Diferencias estadísticamente significativas $\mathrm{p}<0,1 ;(* *): \mathrm{p}<0,05 ;(* * *): \mathrm{p}<0,01$. 
Por lo que respecta a las variables de control - tamaño, sector, tipo de control, antigüedad y estrategia-, se observa que todas ellas, con la excepción del tipo de control de la empresa, resultaron ser significativas. En cuanto a la influencia en el éxito competitivo de las variables explicativas del modelo, se encuentra que la regresión tiene varios coeficientes significativos.

\section{Cuadro 5: Efecto de los factores competitivos en el éxito de las PYME del estado de Veracruz ${ }^{1}$}

\begin{tabular}{|c|c|}
\hline Variables independientes & Beta $^{2}$ \\
\hline - Tamaño & $\begin{array}{l}-0,82 * * * \\
(0,305)\end{array}$ \\
\hline - Sector & $0,141^{* * *}$ \\
\hline - Tipo de control & $\begin{array}{l}(0,267) \\
0,018\end{array}$ \\
\hline - Antigüedad & $\begin{array}{l}(0,309) \\
0,081 * * * \\
(0259)\end{array}$ \\
\hline - Estrategia & $\begin{array}{l}0,067 * * \\
(0,310)\end{array}$ \\
\hline - Capacidades financieras & $\begin{array}{l}0,099 * * * \\
(0,039)\end{array}$ \\
\hline - Posición tecnológica & $\begin{array}{l}0,198 * * * \\
(0,283)\end{array}$ \\
\hline - Innovación & $\begin{array}{l}0,073 * * \\
(0,136)\end{array}$ \\
\hline - $\quad$ Capacidades de marketing & $\begin{array}{l}0,365 * * * \\
(0,050)\end{array}$ \\
\hline - Calidad del producto o servicio & $\begin{array}{l}-0,014 \\
(0,286)\end{array}$ \\
\hline - $\quad \mathrm{RRHH}$ & $\begin{array}{l}0,102 * * * \\
(0,083)\end{array}$ \\
\hline - Formación del gerente & $\begin{array}{l}-0,033 \\
(0,277)\end{array}$ \\
\hline - $\mathrm{TIC}$ & $\begin{array}{l}0,051^{*} \\
(0,046)\end{array}$ \\
\hline $\mathrm{R}^{2}$ & 0,395 \\
\hline $\mathrm{R}^{2}$ corregida & 0,388 \\
\hline $\mathrm{F}$ & $58,819 * * *$ \\
\hline
\end{tabular}

${ }^{1}$ Error estándar entre paréntesis. ${ }^{2}$ Coeficientes estandarizados $(*)$ : Diferencias estadísticamente significativas $(*): \mathrm{p}<0,1 ;(* *): \mathrm{p}<0,05 ;(* * *): \mathrm{p}<0,01$.

Concretamente, las variables capacidades financieras, posición tecnológica, innovación, capacidades de marketing, dirección y gestión de los recursos humanos, así como las tecnologías de la información y comunicación presentan coeficientes de regresión positivos, por lo que cabe asegurar que dichos factores explican el éxito competitivo de las PYME del estado de Veracruz. 
Por otro lado, las variables calidad del producto o servicio y formación del gerente no explican variaciones en la variable dependiente. Los resultados nos llevan a aceptar las hipótesis $\mathrm{H}_{1}, \mathrm{H}_{2}, \mathrm{H}_{3}, \mathrm{H}_{4}, \mathrm{H}_{6} \mathrm{y} \mathrm{H}_{8}$.

Una de las primeras conclusiones que se pueden extraer del examen de los resultados obtenidos es que éstos se ven condicionados por el tamaño, por el sector de actividad y por la antigüedad de la empresa. Además, aquellas PYME con estrategias exploradoras tienen mayores niveles de éxito competitivo.

La existencia de una relación negativa entre el tamaño y el éxito competitivo nos indica que las PYME con menos de 10 trabajadores presentan mayores niveles de competitividad y éxito, lo que puede deberse a su mayor capacidad y velocidad de respuesta a los cambios del entorno. No obstante, este resultado no clarifica demasiado el amplio debate existente en la literatura acerca de la relación entre tamaño y competitividad, dado que muestra resultados contradictorios (Acar, 1993; Wijewardena y Cooray, 1995; Gadenne, 1998).

Más importante es, pues, que el sector influya en los resultados. Este resultado nos indica que pertenecer la empresa al sector industrial tiene mejores resultados que la de servicio. Por otra parte, este resultado también nos confirma la influencia que sobre el éxito competitivo tienen, además de las variables internas, otras relacionadas con el sector de actividad, lo que nos lleva a afirmar que sería interesante establecer un modelo de competitividad de las PYME que conjugara los supuestos propuestos por la teoría de recursos y capacidades con los supuestos más importantes de la economía industrial.

Con referencia a los resultados obtenidos para la variable antigüedad podemos indicar que se ha confirmado la influencia del efecto experiencia. Las PYME que desarrollan su actividad más de 10 años alcanzan mayores niveles de éxito.

Por último, tal y como era de esperarse, se ha puesto de manifiesto la alta dinamicidad del entorno en el que actualmente opera la PYME. Este hecho hace que las PYME con estrategias exploradoras alcancen mayores niveles de éxito. Por tanto, realizar cambios y mejoras en los productos y mercados con relativa frecuencia, tratando de ser la primera en desarrollar nuevos productos, aun con el riesgo de que estas innovaciones no tengan éxito, tiene una clara influencia en la competitividad. 
Los resultados obtenidos para el resto de variables independientes son acordes con los de la bibliografía revisada, con la excepción de las variables calidad del producto o servicio y formación del gerente como indicador de sus capacidades directivas.

En primer lugar, que la calidad del producto y servicio no sea un factor que explique el éxito competitivo puede ser como consecuencia de la mayor inversión que en el corto plazo va a suponer para la empresa la certificación de la calidad, hecho que puede influir en la posición competitiva de la empresa a corto plazo. Un análisis de corte longitudinal pondría de manifiesto la importancia y la necesidad de lograr la satisfacción de los clientes con las características y el diseño de los productos.

En segundo lugar, que la mayor cualificación del gerente no explique el éxito competitivo, no deja de ser un resultado paradójico; en todo caso, a diferencia de lo que indican Wiersema y Bantel (1992), Bantel y Jackson (1989) y Norburn y Birley (1988), se pone de manifiesto que en este caso la calificación del directivo puede que no sea un buen indicador de la capacidad directiva, por lo que para futuras investigaciones habría que tener en cuenta medidas que incorporen una mayor variedad de aspectos.

Con respecto a las capacidades financieras, se ha puesto de manifiesto que el mantenimiento de buenos estándares financieros a través de una adecuada gestión financiera es una de las ventajas competitivas encontradas como clave de éxito en diversos autores (Huck y McEwen, 1991; Viedma, 1992;Acar, 1993; Yusuf, 1995) y en este trabajo. Concretamente, estarán más cerca del éxito competitivo las PYME que realicen, implanten y controlen sistemas de contabilidad de costos, establezcan presupuestos de ingresos y gastos de forma anual y analicen la situación económico-financiera de su empresa.

Por otra parte, los retos competitivos exigen que las empresas introduzcan constantemente innovaciones en los productos y procesos de la empresa, resultados similares a los obtenidos por Viedma (1992), Pil y Holwelg (2003), entre otros. Las PYME deben aprovechar sus ventajas para adaptarse mejor que las grandes a la creación y desarrollo de innovaciones (Ribeiro, 2003) y deben explorar nuevas tecnologías para así encontrar nuevos nichos en el mercado (Pil y Holwelg, 2003).

En lo relativo a la posición tecnológica las PYME para alcanzar el éxito, además de invertir en stocks de tecnologías y en medios humanos y técnicos para su desarrollo, deben contar con información sobre la posición tecnológica de sus com- 
petidores con el fin de desarrollar o adquirir la tecnología que le permita posicionarse por delante de ellos. Los resultados de nuestra investigación son similares a los de Cunninham (1995) y Pil y Holwelg (2003), quienes ponen de manifiesto que contar con una buena posición tecnológica mejora la posición relativa y los resultados de las organizaciones.

Por lo que respecta a las capacidades de marketing, al igual que obtienen en sus investigaciones Pelham (1997 y 2000), Pil y Holwelg (2003) y Verhess y Meulenberg (2004), muestran una clara influencia en el éxito competitivo de las PYME; cabe pensar que es como consecuencia de aprovechar su mayor proximidad al mercado $\mathrm{y}$ al cliente.

El siguiente de los factores de éxito con relación significativa y positiva con la competitividad es el relacionado con la gestión y dirección de los recursos humanos. Además de su importancia para el éxito competitivo, avalada por una gran cantidad de trabajos resaltando, entre ellos, los de Huck y McEwen (1991), Viedma (1992), Reid et al. (2000) y McLarty (2000), es uno de los factores cuya gestión inadecuada se constituye en la principal causa de fracaso de las PYME (McEvoy, 1984).

Por último, de manera similar a los resultados obtenidos por Viedma (1992) y Donrrosoro (2001), nuestro trabajo pone de manifiesto la importancia de contar con sistemas de información que generen autonomía, aumenten el aprendizaje, ayuden a entender mejor el entorno y faciliten la toma de decisiones. Por otro lado, el acceso y uso de las nuevas tecnologías ligadas a internet les puede permitir competir en un mercado cada vez más abierto y competitivo.

\section{CONCLUSIONES}

Con el objetivo de responder a dos cuestiones claves de la investigación de la dirección estratégica, ¿qué se entiende por competitividad? y ¿qué factores internos la propician?, en este trabajo se ha realizado una revisión de los trabajos más relevantes en esta materia.

Contestar a la primera de las cuestiones no es fácil. La existencia de diferentes unidades, marcos teóricos y trabajos empíricos dificultan la elección de una definición clara y operativa. Hemos aportado una conceptualización clara al definir la 
competitividad como la capacidad de la empresa para, rivalizando con otras empresas, conseguir y alcanzar una posición competitiva favorable que permita obtener un desempeño superior al de los competidores. Además, se operativiza el concepto revisando para ello diversas investigaciones empíricas. Como principal aportación de esta revisión, resalta la importancia de utilizar un indicador global de éxito que incluye diferentes aspectos de la competitividad, tanto cuantitativos como cualitativos, y la utilización de fuentes subjetivas para permitir conocer la posición relativa de la empresa frente a la competencia.

Una vez resuelta la primera de las cuestiones, y desde el marco de la teoría de recursos y capacidades, se han encontrado diversos factores que la bibliografía especializada reconoce como estratégicos para las PYME. Concretamente, el éxito competitivo de las empresas de menor tamaño se explica por la capacidad financiera, por los recursos tecnológicos, por la innovación, por las capacidades de marketing, por la calidad del producto o servicio, por la dirección de recursos humanos, por las capacidades directivas para gestionar la empresa y por la intensidad de las tecnologías de la información y comunicación.

Para contrastar estos planteamientos, se ha utilizado una muestra de 1.425 PYME, representativas del estado de Veracruz, encontrando que las variables de control (tamaño, sector, antigüedad y tipo de estrategia) son factores que explican parcialmente el éxito competitivo de las PYME.

Por otro lado, el contraste de las hipótesis realizado permite resaltar varios factores como explicativos del éxito competitivo de las PYME; nos referimos a las capacidades financieras, la posición tecnológica, la innovación, las capacidades de marketing, la dirección de los recursos humanos y las tecnologías de la información y comunicación.

Estos resultados indican la necesidad de gestionar adecuadamente los recursos financieros dado que su inadecuada gestión puede comprometer, por la gran debilidad de los balances en las empresas de menor tamaño, además del éxito competitivo la propia supervivencia de la empresa.

Por otra parte, es claro que las PYME que quieran mejorar su posición competitiva frente a la competencia deberán adquirir o desarrollar nuevos activos tecnológicos. Si los principales competidores tienen una tecnología más eficiente y moderna o las organizaciones sólo realizan inversiones al comprobar los buenos resultados obtenidos por las empresas rivales, será menor su capacidad para competir. 
Asimismo, estos nuevos retos competitivos exigen que las empresas analicen constantemente las mejoras que pueden ir introduciendo en sus procesos y productos. La innovación continua es uno de los factores clave para atender las necesidades cambiantes de los clientes y por tanto se constituye como un factor decisivo para el éxito competitivo de las PYME.

Además, las PYME de éxito se caracterizan por una clara orientación hacia el cliente y el mercado al resaltar la importancia de las capacidades de marketing; también se pone de manifiesto la importancia que tiene la gestión y dirección de los recursos humanos. Las PYME deben prestar gran atención a aquellas prácticas que favorezcan el desarrollo del capital humano y que permitan gestionar correctamente el conocimiento organizativo.

El último de los factores significativos es el relacionado con la intensidad de las tecnologías de la información y comunicación. La evolución hacia la sociedad de la información y el conocimiento hace que las tecnologías relacionadas con ella adquieran una gran importancia para competir. Las empresas que quieran obtener ventajas sobre la competencia deberán acceder e implantar tecnologías que permitan obtener, procesar y transmitir grandes volúmenes de información a cada vez más lugares y en el menor tiempo posible.

A modo de síntesis, es posible afirmar que los resultados obtenidos son en muchos aspectos consistentes con la literatura, aunque quedan abiertas otras cuestiones en las que se ha de seguir profundizando como son la explicación de la influencia del tipo de control de la empresa, de la calidad del productos o servicio y la cualificación del gerente.

Asimismo, es necesario señalar algunas de las limitaciones encontradas en la realización de este trabajo. A las propias de la medida de éxito utilizada, hay que sumar que el estudio planteado sea de corte transversal, hecho que imposibilita el examen de las relaciones del éxito y los factores que lo explican a lo largo del tiempo.

No obstante, se considera que con este trabajo se hace una importante aportación al estudio de los factores explicativos del éxito competitivo de las PYME en el contexto de México, si bien el trabajo se concentra en el estudio de las PYME del estado de Veracruz, quedando pendiente la ampliación del modelo tanto a nuevos estados como a la incorporación de nuevas variables de naturaleza más intangible que se consideren relevantes para la competitividad y el éxito del colectivo de empresas estudiadas. 


\section{BIBLIOGRAFÍA}

Acar, A. (1993): "The impact of key internal factors on firms performance: An empirical study of small Turkish firms", Journal of Small Business Management, October, Vol. 31, N. 4, pp.86-92.

Ahiere, S.L.; Golhar, D.Y. (1996): “Quality management in large versus small firms”, Journal of Small Business Management, vol.34, n.2, pp.1-11.

Álvarez, J.C.; García, E. (1996): "Factores de éxito y riesgo en la pyme: Diseño e implantación de un modelo para la mejora de la competitividad", Economía Industrial, N. 310, pp. 149-161.

Bantel, K.A.; Jackson, S.E.(1989): “Top management and innovations in banking: does the composition of the top team make the difference?", Strategic Management Journal, Vol.10, pp.107-124.

Birley, S.; Westhead, P. (1990): "Growth and performance contrasts between types of small firms", Strategic Management Journal, Vol.11, N.7, pp.535-557.

Camelo, C.; Lorenzo, J.D.; Martín, F.; Valle, R. (1999): “Competitividad regional y recursos intangibles: Un análisis comparado Andalucía-España”, Servicio de Publicaciones de la Universidad de Cádiz, Coordinado por Ruiz, J.

Camisón, C. (1997): La competitividad de la PYME industrial española: estrategia y competencias distintivas, Cívitas, Madrid.

(1999): "La medición de los resultados empresariales desde una óptica estratégica: construcción de un instrumento a partir de un estudio Delphi y aplicación a la empresa industrial española en el periodo 1983-1996", Revista de Contabilidad y Tributación, Centro de Estudios Financieros, N. 199, pp. 201-264.

(2001): La competitividad de la empresa industrial de la Comunidad Valenciana: análisis del efecto del atractivo del entorno, los distritos industriales y las estrategias empresariales, Editorial Tirant lo Blanch, Valencia. 
Chang, S.; Singh, H. (2000): "Corporate and industry effects on business unit competitive position”, Strategic Management Journal, Vol.21, N.7, pp.739752 .

Churchill, G.A. (1979): “A paradigm for developing better measures of marketing constructs”, Journal of Marketing Research, February, vol.16, pp.64-73.

Clifford, D.K. Jr.; Cavanagh, R.E. (1985): The winning performance: how America's high-growth midsize companies succeed, Utilizada la versión en español Estrategias de éxito para la pequeña y mediana empresa, Ediciones Folio, 1989.

Conant, J.S.; Mokwa, M.P.; Varadarajan, P.R. (1990): “Strategic Types, distinctive marketing competencies and organizational performance: a multiple measuresbased study", Strategic Management Journal, Vol. 11, pp. 365-383.

Covin, J.G.; Prescott, J.E.; Slevin, D.P. (1990): "The effects of technological sophistication on strategic profiles, structure and firm performance", The Journal of Management Studies, September, Vol.27, N.5, pp.485-510.

Cunningham, J.B. (1995): "Success in corporate and entrepreneurial organizations in Singapore", Journal of Small Business Management, Vol. 33, N. 4, October, pp. 80-86.

Daily, C.M.; Dalton, D.R. (1992): "Financial performance of founder- managed versus professionally managed small corporations", Journal of Small Business Management, April, Vol.30, N.2, pp.25-34.

Dess G.D.; Robison, R.B. Jr. (1984): "Measuring Organizational Performance in de absence of objective measures: the case of Privately-held firm and conglomerate business unit", Strategic Management Journal, Vol. 5, pp. 265-273.

Dollinguer, M.J. (1984): "Enviromental boundary spanning and information processing effects on organizational performance", Academy of Management Journal, Vol.27, N.2, pp.351-368. 
Donrrosoro, I.; García C.; González, M; Lezámiz, M.; Matey, J.; Moso, M.; Unzuela, M. (2001): "El modelo de gestión de las PYMEs vascas de éxito", Edita Cluster del Conocimiento (Portal de Gestión) y ediciones PMP, Bilbao.

Gadenne, D. (1998): "Critical success factors for small business: An inter-industry comparison", International Small Business Journal, Oct-Dec, Vol. 17, Issue 1, pp. 36-56.

Galán, J.L.; Vecino, J. (1997): "Las fuentes de rentabilidad de las empresas", Revista Europea de Dirección y Economía de la empresa, Vol. 6, N. 1, pp. 21-36.

Gomez-Mejia, Luis R.; Balkin, David B.; Cardy, Robert L. (1996): Gestión de Recursos Humanos, Prentice Hall, Madrid.

Grant, R. M. (1996): Dirección Estratégica: Conceptos, Técnicas y Aplicaciones, Cívitas, Madrid.

Hair, J.F.; Anderson, R.E.; Tatham, R.L.; Black, W.C., (1999): Análisis multivariante, Prentice Hall, Madrid.

Huerta, E. (ed.); Bayo, J.A.; García, C.; Merino, J. (2003): Los desafíos de la competitividad. La innovación organizativa y tecnológica en la empresa española, Fundación BBVA, Bilbao.

Huang, T. (2001): "The relation of training practices and organizational performance in small and medium size enterprises", Education \& Training, Vol.43, N.8/9, pp.437-444.

Huck, J.; McEwen, T. (1991): "Competencies needed for small business success: Perceptions of Jamaican entrepreneurs", Journal of Small Business Management, October, Vol. 29, N. 2, pp. 90-93.

Jennings, P.; Beaver, G. (1997): "The performance and competitive advantage of small firms: a management perspective", International Small Business Journal, January-February, Vol.15, N.2, pp.63-73. 
Kalleberg, A.L.; Leitcht, K.T. (1991): “Gender and Organisational Performance: Determinants of Small Business Survival and Success", Academy of Management Journal, Vol.34, pp.136-161.

Kay, J. (1993): Foundations of corporate success, Oxford University Press. Utilizada versión traducida (1994): Fundamentos del éxito empresarial, Ariel Sociedad Económica.

Kester, C.; Luehrman, T. (1989): "Are we feeling more competitive yet? The exchange rate gambit”, Sloan Management Review, Winter, N. 19, pp. 19-28.

Kirby, D.A. (1990): "Management education and small business development: an exploratory study of small firms in de U.K.", Journal of Small Business Management, October, Vol.28, N.4, pp.78-87.

Kotey, M.; Meredith, G.G. (1997): "Relationships among owner/manager personal values, business strategies, and enterprise performance", Journal of Small Business Management, April, Vol.35, N.2, pp.37-61.

Lin, C. (1998): "Success factors of small -and medium- sized enterprise in Taiwan: An analysis of cases", Journal of Small Business Management, October, Vol. 36, N. 4, pp. 43-56.

Loan-Clarke, J.; Boocock, G.; Smith,A. Whittaker, J. (1999): “Investment in training and development by small business", Employee Relations, Vol.21, N.3, p.296301.

Luck, S. (1996): "Success in Hong Kong: Factors self-reported by successful small business owners", Journal of Small Business Managemnet, October, Vol. 34, N. 3, pp. 68-75.

Llopis, J. (2000): Dirigiendo: 11 factores clave del éxito empresarial, Ediciones Gestión 2000, Barcelona.

Marbella, F. (1998): “Competitividad de las empresas Castellano-Leonesas: Análisis de algunos factores relevantes", Sexto Congreso de Economía Regional de Castilla y León, Zamora. 
Mauri, A.; Michaels, M. (1998): "Firm and Industry effects within strategic management: an empirical examination", Strategic Management Journal, Vol. 19, pp. 211-219.

McGahan, A. (1999): “The performance of US corporations: 1981-1994”, Journal of industrial economics, December, Vol. XLVII, N. 4.

McGahan, A.; Porter, M. (1997): "How much does industry matter, really?", Strategic Management Journal, Vol. 18 (Summer Special Issue), pp. 15-30.

McLarty, R. (2000): "Evaluating graduate skills in SMEs: the value chain impact", The Journal of Management Development, Vol.19, N.7, pp.615-628.

Molina, J.F. (2003): Factores determinantes de la rentabilidad empresarial en la Comunidad Valenciana: Un análisis de la importancia de los efectos empresa, industria y territorio, Publicaciones de la Universidad de Alicante, Alicante.

Nelson, R. (1992): "Recent writings on competitiveness: boxing the compass", California Management Review, Winter, pp. 127-37.

Norburn, D.; Birlry, S. (1988): "The top management team and corporate performance", Strategic Management Journal, May/June, Vol.9, pp.225-237.

Ottewill, P.; Jennins, P.; Magirr, P. (2000): "Management competence development for professional service SMEs: the case of community pharmacy",Education \& Trainning, Vol.42, N.4/5, pp.246-254.

Paig, R.C.; Littrell, M.A. (2002): "Craft Retailers' criteria for success and associated business strategies", Journal of Small Business Management, Vol.40, N.4, pp.314-331.

Pelham, A.M. (1997): "Mediating influences on the relationship between market orientation and profitability in small industrial firms", Journal of Marketing Theory and Practice, Summer, vol.5, n.3, pp.55-76.

(2000): "Market Orientation and other potential influences on performance in small an medium-sized manufacturing firms", Journal of Small Business Management, Vol.38, N.1, pp.48-67. 
Peters, T.; Waterman, R. (1982): In search of excellence, Nueva York: Harper \& Row. Utilizada la versión española En busca de excelencia, Ediciones Folio, 1992.

Pfeffer, J. (1994): “Competitive advantage trough people”, California Management Review, N.36, pp. 9-29.

Pil, F.; Holwelg, M. (2003): “The advantages of thinking small”, Mit Sloan Management Review, Winter, pp.33-39.

Porter, M. (1990): The competitive advantage of nations, New York, The Free Press Word Economic Forum. Traducción (1991): La ventaja competitiva de las naciones, Plaza \& Janes. Barcelona.

Powell, T.C. (1996): "How much industry matter? An alternative empirical test", Strategic Management Journal, Vol. 17, pp. 323-334.

Puig, P. (1996): La competitivitat de l'empresa industrial a Catalunya. Anàlisis de l'entorn econòmic $i$ de las estratègies competitives en un contexte de modernització del sector públic, ESADE, Barcelona.

Ribeiro, D. (2003): "Rendimiento de las pymes innovadoras", Revista Europea de Dirección y Economía de la Empresa, Vol.12, N.3, pp.119-132.

Rogoff, E.G.; Lee, M.; Suh, D. (2004): "Who done it? Attributions by entrepreneurs and experts of the factors that cause and impede small business success", Journal of Small Business Management, Vol.42, N.4, pp. 374-376.

Roquebert, J.; Phillips, R.; Westfall, P. (1996): "Markets vs. Management: what drives profitability?", Strategic Management Journal, vol.17, pp.653-664.

Rumelt, R. (1991): “How much industry matter?”, Strategic Management Journal, Vol. 12, pp. 167-185.

Salomon, G.L. (1985): “Accounting rates of return”, The American Economic Review, Vol.5, N.3, pp.495-504. 
Santos, M.V.; García, M.T.; Pérez, M.P. (1998): “Adaptación e imitación en la estrategia empresarial. El caso de la estructura de mercado de las cajas de ahorro españolas", Cuadernos de economía y dirección de empresas, Vol. 2, pp. 203-230.

Santos, L.; Gonzalez, O. (2000): "Economic success factors in Spanish small retail businesses”, Small Business Economics, Vol.15, pp.209-222.

Schmalensee, R. (1985): "Do Markets Differ Much?", The American Economic Review, June, N. 3, pp. 341-351.

Smith, A.; Whittaker, J.; Clark, J.W.; Boocock, G. (1999): “Competence based management development provision to SMEs and the providers perspective", The Journal of Management Development, Vol.18, N.6, pp.557-563.

Varadajan, P. R.; Ramanujam, V. (1990): "The corporate performance conundrum: a synthesis of contemporary views and an extension", Journal of Management Studies, September, Vol.27, N.5, pp.463-483.

Venkatram, N.; Ramanujam, V. (1986): "Measurement of business performance in strategy research", Academy of Management Review, Vol. 11, N. 4, pp. 801-814.

Verhees, F.J.H.M.; Meulenberg, M.T.G. (2004): "Market orientation, innovativeness, product innovation, and performance in small firms", Journal of Small Business Management, Vol.42, N.2, pp. 86-91.

Viedma, J.M. (1992): La excelencia empresarial española, Mc Graw Hill. Segunda Edición.

Vickerstaff, S. (1992): "The management training in the smaller firm", Target Management Development Review, Vol.5, N.4, pp.32-36.

Vinten, G. (2000): "Training in small-and medium-sized enterprises", Industrial and Commercial Training, Vol.32, N.1, pp.1-9. 
Warren, L.; Hutchinson, W. (2000): "Success factors for high-technology SMEs: A case Study from Australia", Journal of Small Business Management, July, pp. 86-91.

Wernerfelt, B.; Montgomery, C. (1988): “Tobin's q and the importance of Focus in Firm Performance", The American Economic Review, Vol. 78, N. 1, pp. 246250 .

Wiersema, M.F.; Bantel, K.A. (1992): "Top management team demography and corporate strategic change", Academy of Management Journal, Vol.35, N.1, pp.91-121.

Wijewardena, H.; Cooray, S. (1995): "Determinants of growth in small Japanese manufacturing firms survey evidence from Kobe", Journal of Small Business Management, October, Vol. 33, N. 4, pp. 87-92.

Yusuf,A. (1995): "Critical success factors for small business: perceptions of South Pacific entrepreneurs", Journal of Small Business Management, April, Vol. 33, N.2, pp. 68-73. 